\title{
Metformin prevents ischaemic ventricular fibrillation in metabolically normal pigs
}

\author{
Li Lu ${ }^{1,2} \cdot$ Shuyu Ye ${ }^{1,2}$ - Rebecca L. Scalzo ${ }^{2,3}$. Jane E. B. Reusch ${ }^{2,3}$. \\ Clifford R. Greyson ${ }^{1,2}$ • Gregory G. Schwartz ${ }^{1,2}$
}

Received: 25 December 2016/Accepted: 29 March 2017 / Published online: 11 May 2017

(C) Springer-Verlag (outside the USA) 2017

\begin{abstract}
Aims/hypothesis Metformin is the drug most often used to treat type 2 diabetes. Evidence suggests that metformin may reduce mortality of individuals with type 2 diabetes, but the mechanism of such an effect is unknown and outcomes of metformin treatment in people without diabetes have not been determined. If metformin favourably affected mortality of non-diabetic individuals, it might have even broader therapeutic utility. We evaluated the effect of metformin on myocardial energetics and ischaemic ventricular fibrillation (VF) in metabolically normal pigs.

Methods Domestic farm pigs were treated with metformin (30 $\mathrm{mg} \mathrm{kg}^{-1}$ day $^{-1}$ orally for $2-3$ weeks; $n=36$ ) or received no treatment $(n=37)$. Under anaesthesia, pigs underwent up to $90 \mathrm{~min}$ low-flow regional myocardial ischaemia followed by $45 \mathrm{~min}$ of reperfusion. Pigs were monitored for arrhythmia, monophasic action potential morphology, haemodynamics and myocardial substrate utilisation, AMP-activated protein kinase (AMPK) phosphorylation activity and ATP concentration.

Results Death due to VF occurred in $12 \%$ of pigs treated with metformin compared with $50 \%$ of untreated controls
\end{abstract}

Electronic supplementary material The online version of this article (doi:10.1007/s00125-017-4287-2) contains peer-reviewed but unedited supplementary material, which is available to authorised users.

Gregory G. Schwartz

Gregory.Schwartz@va.gov

Cardiology Section, Denver VA Medical Center, 1055 Clermont St, Mail Code 111B, Denver, CO 80220, USA

2 University of Colorado School of Medicine, Aurora, CO, USA

3 Endocrinology/Metabolism Section, Denver VA Medical Center, Denver, CO, USA $(p=0.03)$. The anti-fibrillatory effect of metformin was associated with attenuation of action potential shortening in ischaemic myocardium $(p=0.02)$ and attenuation of the difference in action potential duration between ischaemic and non-ischaemic regions $(p<0.001)$ compared with untreated controls. Metformin had no effect on myocardial contractile function, oxygen consumption, or glucose or lactate utilisation. During ischaemia, however, metformin treatment amplified the activation of AMPK and preserved ATP concentration in myocardium compared with untreated controls (each $p<0.05$ ).

Conclusions/interpretation Chronic treatment of metabolically normal pigs with metformin at a clinically relevant dose reduces mortality from ischaemic VF. This protection is associated with preservation of myocardial energetics during ischaemia. Maintenance of myocardial ATP concentration during ischaemia is likely to prevent action potential shortening, heterogeneity of repolarisation, and propensity for lethal arrhythmia. The findings suggest that metformin might be protective in non-diabetic individuals with coronary heart disease.

Keywords Action potential · ATP · Ischaemia $\cdot$ Metformin • Ventricular fibrillation

$\begin{array}{ll}\text { Abbreviations } \\ \text { AMPK } & \text { AMP-activated protein kinase } \\ \text { CS } & \text { Citrate synthase } \\ \mathrm{K}_{\text {ATP }} & \text { ATP-sensitive potassium } \\ \text { LAD } & \text { Left anterior descending coronary artery } \\ \text { LV } & \text { Left ventricle } \\ \text { MAP } & \text { Monophasic action potential } \\ \text { VF } & \text { Ventricular fibrillation }\end{array}$




\section{Introduction}

The United Kingdom Prospective Diabetes Study (UKPDS) demonstrated a reduction in mortality among individuals with type 2 diabetes who were treated with metformin compared with usual care [1]. Subsequent observational data and meta-analyses supported this finding [2-5]. Today, more than 100 million individuals with type 2 diabetes are treated with metformin worldwide [6]. However, it is uncertain how metformin reduces mortality in people with diabetes, and it is unknown whether metformin has the potential to reduce mortality in people without diabetes. If so, metformin might have therapeutic utility in an even broader range of people than those presently treated with the drug.

Treatment with metformin did not improve left ventricular (LV) function after myocardial infarction [7] or attenuate progression of carotid atherosclerosis [8] in non-diabetic individuals. However, metformin improved survival after experimental myocardial infarction in rats, even though there was no effect of treatment on infarct size or LV function [9]. In aggregate, these findings raise the possibility that mechanisms other than preservation of ischaemic myocardium or attenuation of atherosclerosis could be responsible for any reduction of mortality that might result from metformin treatment in the absence of diabetes.

No prior study has investigated the effects of metformin on ischaemic cardiac arrhythmias and sudden cardiac death. There is evidence that metformin favourably influences myocardial ATP concentration in rodents $[9,10]$ and that an inverse relationship exists between myocardial ATP concentration and risk of ischaemic ventricular fibrillation (VF) $[11,12]$. Against this background, we tested the hypothesis that metformin reduces ischaemic VF in association with favourable effects on cardiac energetics in metabolically normal pigs.

\section{Methods}

Pigs and treatments Protocols were approved by the Institutional Animal Care and Use Committees of the University of Colorado and the Denver VA Medical Center and were conducted in accordance with the Guide for the care and use of laboratory animals published by the National Institutes of Health. Experiments were performed in a total of 92 Yorkshire cross breed pigs of either sex (K\&S Livestock, Ft Collins, CO, USA) weighing $25 \pm 1 \mathrm{~kg}$ and provided with standard laboratory housing and a chow diet. Treatments and measurements are summarised in the electronic supplementary material (ESM). Metformin for chronic, oral treatment was administered as $500 \mathrm{mg}$ pharmaceutical tablets (Major Pharmaceuticals, Livonia, MI, USA). The tablets were hand-fed to pigs $(n=36)$ in a cookie at a dosage of 30
Table 1 Haemodynamics, MAP duration and substrate uptake at baseline and during ischaemia

\begin{tabular}{|c|c|c|}
\hline Treatment group & $\begin{array}{l}\text { Chronic } \\
\text { metformin }\end{array}$ & $\begin{array}{l}\text { Untreated } \\
\text { controls }\end{array}$ \\
\hline \multicolumn{3}{|c|}{ LV systolic pressure, $\mathrm{mmHg}$} \\
\hline Baseline & $105 \pm 5$ & $96 \pm 3$ \\
\hline $15 \mathrm{~min}$ ischaemia & $93 \pm 4 *$ & $90 \pm 4$ \\
\hline \multicolumn{3}{|l|}{ Heart rate (bpm) } \\
\hline Baseline & $109 \pm 1$ & $107 \pm 3$ \\
\hline $15 \mathrm{~min}$ ischaemia & $108 \pm 1$ & $105 \pm 2$ \\
\hline \multicolumn{3}{|c|}{ LAD blood flow (ml/min) } \\
\hline Baseline & $17 \pm 2$ & $20 \pm 2$ \\
\hline $15 \mathrm{~min}$ ischaemia & $8 \pm 1 * * *$ & $10 \pm 1 * * *$ \\
\hline \multicolumn{3}{|l|}{ MAP duration (ms) } \\
\hline Baseline & $310 \pm 8$ & $305 \pm 7$ \\
\hline $15 \mathrm{~min}$ ischaemia & $297 \pm 11^{\dagger}$ & $263 \pm 7 *$ \\
\hline \multicolumn{3}{|l|}{$\mathrm{LV} \mathrm{dP} / \mathrm{dt}_{\max }(\mathrm{mmHg} / \mathrm{s})$} \\
\hline Baseline & $2006 \pm 147$ & $1984 \pm 132$ \\
\hline $15 \mathrm{~min}$ ischaemia & $1678 \pm 107 * * *$ & $1646 \pm 87^{*}$ \\
\hline \multicolumn{3}{|c|}{ LV regional stroke work index $(\mathrm{kPa})$} \\
\hline Baseline & $4.0 \pm 0.3$ & $3.7 \pm 0.3$ \\
\hline $15 \mathrm{~min}$ ischaemia & $1.7 \pm 0.1 * * *$ & $1.8 \pm 0.2 * * *$ \\
\hline \multicolumn{3}{|c|}{$\begin{array}{l}\text { Coronary arteriovenous oxygen } \\
\text { extraction }(\mu \mathrm{mol} / \mathrm{ml})\end{array}$} \\
\hline Baseline & $3.9 \pm 0.2$ & $3.7 \pm 0.2$ \\
\hline $15 \mathrm{~min}$ ischaemia & $4.0 \pm 0.2 *$ & $4.3 \pm 0.2 *$ \\
\hline \multicolumn{3}{|c|}{$\begin{array}{l}\text { Coronary arteriovenous glucose } \\
\text { extraction }(\mu \mathrm{mol} / \mathrm{ml})\end{array}$} \\
\hline Baseline & $0.04 \pm 0.03$ & $0.22 \pm 0.15$ \\
\hline $15 \mathrm{~min}$ ischaemia & $0.94 \pm 0.18 * * *$ & $1.14 \pm 0.11 * *$ \\
\hline \multicolumn{3}{|c|}{$\begin{array}{l}\text { Coronary arteriovenous lactate } \\
\quad \text { extraction }(\mu \mathrm{mol} / \mathrm{ml})\end{array}$} \\
\hline Baseline & $0.41 \pm 0.50$ & $0.39 \pm 0.47$ \\
\hline $15 \mathrm{~min}$ ischaemia & $-1.96 \pm 0.36^{* *}$ & $-2.85 \pm 0.39 * * *$ \\
\hline
\end{tabular}

Data are mean \pm SEM

For haemodynamic measurements: $n=11$ for chronic metformin and $n=10$ for untreated controls; for MAP: $n=13$ each for chronic metformin and untreated controls; for oxygen consumption and arteriovenous glucose and lactate extraction: $n=12$ for chronic metformin and $n=8$ for untreated controls

$* p<0.05, * * p<0.01, * * * p<0.001$ compared with baseline in same group; ${ }^{\dagger} p<0.05$ for difference between chronic metformin treatment and untreated controls

$35 \mathrm{mg} \mathrm{kg}{ }^{-1}$ day $^{-1}$, administered in two divided doses for 2-3 weeks. This dosage is comparable with that used in clinical practice. Metformin for acute, intravenous administration (Sigma Aldrich, St Louis, MO, USA) was dissolved in $100 \mathrm{mg} / \mathrm{ml}$ normal saline $(154 \mathrm{mmol} / \mathrm{l} \mathrm{NaCl})$ and administered to 19 pigs at a dose of $100 \mathrm{mg} / \mathrm{kg}$ i.v. under general anaesthesia (described below). On-treatment measurements were begun 180 min after this dose. Chronic and acute metformin groups were compared with a control group of 37 pigs that 
received no metformin treatment. Each treatment group contained the same proportion of male and female pigs. Treatment assignment was not blinded.

Anaesthesia and instrumentation of the heart Pigs were sedated with intramuscular ketamine and midazolam, anaesthetised with intravenous alpha-chloralose and midazolam, and mechanically ventilated through an endotracheal tube as previously described [13]. LV pressure was measured with a solid state catheter inserted through a carotid artery introducer sheath. An intravenous infusion of normal saline was adjusted to maintain LV end-diastolic pressure at $5-10 \mathrm{mmHg}$. Arterial blood glucose was monitored every $30 \mathrm{~min}$ and maintained at $4.5 \pm 0.5 \mathrm{mmol} / 1$ by adjusting the rate of infusion of $10 \%$ dextrose solution. The chest was opened via median sternotomy and the heart suspended in a pericardial cradle. In experiments involving myocardial ischaemia, an ultrasonic transit time flow probe (Transonic Systems, Ithaca, NY, USA) and hydraulic occluder were placed around the left anterior descending coronary artery (LAD) just distal to its first diagonal branch. In experiments with measurements of coronary arteriovenous concentration differences in oxygen, glucose and lactate, a catheter was placed in the anterior interventricular coronary vein at a position adjacent to the coronary occluder to allow paired sampling of arterial and coronary venous blood. In experiments that assessed regional contractile function, LV pressure-wall area loops were determined as previously described [14] using a quadrangular array of sonomicrometry crystals (Sonometrics, London, ON, Canada) implanted in the subendocardium of the anterior LV. The area of the loops was calculated as an index of regional stroke work.

Low-flow ischaemia and reperfusion Regional, low-flow myocardial ischaemia was induced in 25 pigs in the chronic metformin group, 11 pigs in the acute metformin group and 23 pigs in the untreated group. After baseline measurements, the LAD was constricted with the hydraulic occluder until flow was reduced to $50 \%$ of baseline $( \pm 1 \mathrm{ml} / \mathrm{min})$. In experiments without a myocardial biopsy during ischaemia, coronary flow was maintained at this level for $90 \mathrm{~min}$ or until VF occurred, followed by $45 \mathrm{~min}$ of reperfusion. In experiments where a myocardial biopsy was obtained at $15 \mathrm{~min}$ of ischaemia, the experiment was terminated after the biopsy. We have previously shown that this ischaemic intervention produces significant metabolic and functional impairment without myocardial infarction $[15,16]$.

Ischaemic VF Survival analysis was used to assess freedom from VF during ischaemia and reperfusion. Pigs that underwent myocardial biopsy during ischaemia were excluded from survival analysis because the drill biopsy itself can induce VF. Thus, survival was assessed in 17 pigs in the chronic metformin group, 11 pigs in the acute metformin group and 16 pigs in the untreated group.

Monophasic action potential recording Monophasic action potential (MAP) recording from the epicardial surface of the LV represents the local cardiac action potential and is a technique well suited for in situ studies [17]. MAP was recorded in 13 pigs in the chronic metformin group, 13 untreated control pigs and 11 pigs treated acutely with metformin. A hand-held MAP probe (EP Technologies, Mountain View, CA, USA) was employed as previously described [18]. MAP duration was measured from the initial upward deflection of phase 0 depolarisation to the time of $90 \%$ recovery from the peak of phase 0 . MAP was recorded from the anterior LV (ischaemic region) at baseline, at 15, 30, 60 and 90 min of ischaemia, and at $45 \mathrm{~min}$ of reperfusion, contingent upon survival of pigs to each time point without $\mathrm{VF}$. In four pigs in the chronic metformin group and 13 untreated pigs, MAP was also recorded in a remote, non-ischaemic region of the lateral LV free wall, prior to and during ischaemia of the anterior LV wall.

Myocardial ATP content In eight pigs from the chronic metformin treatment group and seven untreated controls, myocardium from the centre of the ischaemic region was biopsied at $15 \mathrm{~min}$ of ischaemia using a high-speed drill attached to a collection canister under vacuum and filled with isopentane chilled by a jacket of liquid nitrogen. The flash-frozen biopsies were assayed for ATP as previously described [19] with a Biovision K354 fluorometric assay (Biovision, Milpitas, CA, USA).

Interaction of metformin with ATP-sensitive potassium channel opener Ordinarily, cardiac ATP-sensitive potassium $\left(\mathrm{K}_{\text {ATP }}\right)$ channels open during ischaemia, causing the action potential to shorten. To determine whether effects of metformin on ischaemic action potential duration reflect an action of the drug as a direct $\mathrm{K}_{\text {ATP }}$ blocker, eight pigs treated chronically with metformin and 15 untreated controls underwent recording of MAP from the epicardium of the anterior LV in the absence of ischaemia, prior to and during infusion of a $\mathrm{K}_{\text {ATP }}$ opener into the LAD (levcromakalim, $1 \mu \mathrm{g} \mathrm{kg}^{-1} \mathrm{~min}^{-1}$ ) [18]. The presence or absence of $\mathrm{K}_{\mathrm{ATP}}$, blockade by metformin was inferred from whether or not metformin attenuated the MAP shortening induced by levcromakalim.

AMP-activated protein kinase (AMPK), phospho-AMPK$\mathrm{Thr}^{172}$ and citrate synthase activity Myocardial drill biopsy samples from the lateral LV (prior to ischaemia) and from the central portion of the ischaemic region (at $15 \mathrm{~min}$ of ischaemia) were frozen for subsequent western blotting. Total AMPK (no. 2532, 1:1000; Cell Signaling Technology, 
Danvers, MA, USA) and phospho-AMPK-Thr ${ }^{172}$ (no. 2531S, 1:1000; Cell Signaling Technology) were quantified using infrared fluorescence. Citrate synthase (CS) activity was assayed using previously published methods [20, 21].

Statistical analysis Under each experimental condition in each pig, three to five measurements of haemodynamics, two to three measurements of MAP duration, and two measurements of transmyocardial oxygen, glucose and lactate extraction were made and then averaged. Myocardial biopsy was performed once under each experimental condition in each sampled region of the heart. Data were obtained and are reported herein from all pigs that underwent technically successful surgery and instrumentation. Data are presented as mean \pm SEM. For individual measurements, differences between metformin-treated and untreated groups were evaluated by Student's $t$ test. Differences in survival free of VF were assessed by logrank analysis. Two-way ANOVA was used to determine the effect of ischaemia between treatment groups where appropriate. Statistical significance was defined by a $p$ value $<0.05$.

\section{Results}

Haemodynamics and LV function at baseline and during ischaemia At baseline, chronic metformin treatment had no effect on haemodynamics (heart rate, LV systolic pressure, LAD blood flow) or indices of regional and global LV contractile function (regional stroke work, $\mathrm{LV} \mathrm{dP} / \mathrm{dt}_{\max }$ ). During ischaemia, both groups exhibited similar reduction of contractile function, increased transmyocardial oxygen and glucose extraction, and net lactate release (Table 1). Acute metformin treatment also had no effect on haemodynamics or contractile function (data not shown).

Ischaemic VF VF occurred in 50\% of untreated controls (eight out of 16 pigs), predominantly between 10 and 45 min of ischaemia (Fig. 1). Chronic metformin treatment reduced the occurrence of VF to $12 \%$ (two of 17 pigs; $p=0.03$ compared with untreated controls) and significantly improved survival free of VF $(p=0.02)$. Acute metformin treatment had no effect on the occurrence of VF (45\%; five of 11 pigs) compared with untreated controls.

MAP duration MAP was recorded to determine whether the anti-fibrillatory effect of chronic metformin treatment was related to an effect on action potentials during ischaemia. Prior to ischaemia, there was no effect of chronic metformin treatment on MAP duration (Table 1). During ischaemia in untreated pigs, MAP shortened as expected [22] to a nadir at approximately $15 \mathrm{~min}$ of ischaemia and then gradually recovered with continued ischaemia and reperfusion (Fig. 2).

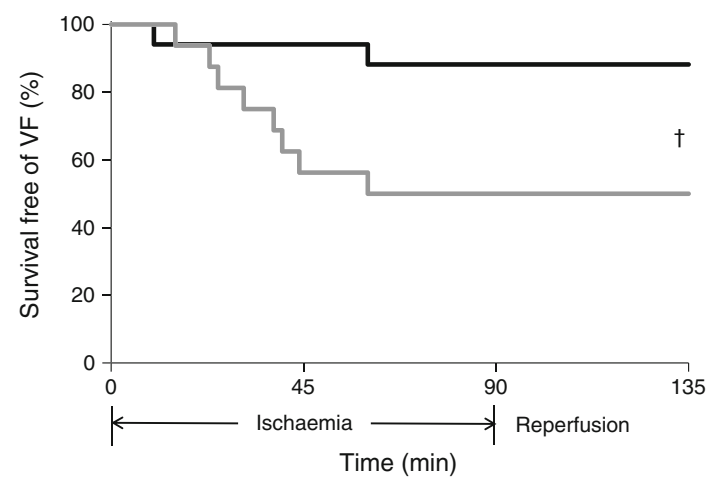

Fig. 1 Survival free of VF during low-flow myocardial ischaemia and reperfusion. Survival free of VF was greater in pigs that received chronic metformin treatment (black, $n=17$ ) compared with untreated controls (grey, $n=16$ ). ${ }^{\dagger} p=0.02$

Placed in context with the data in Fig. 1, there was a temporal correspondence between the nadir of MAP duration and the highest incidence of ischaemic VF in untreated pigs.
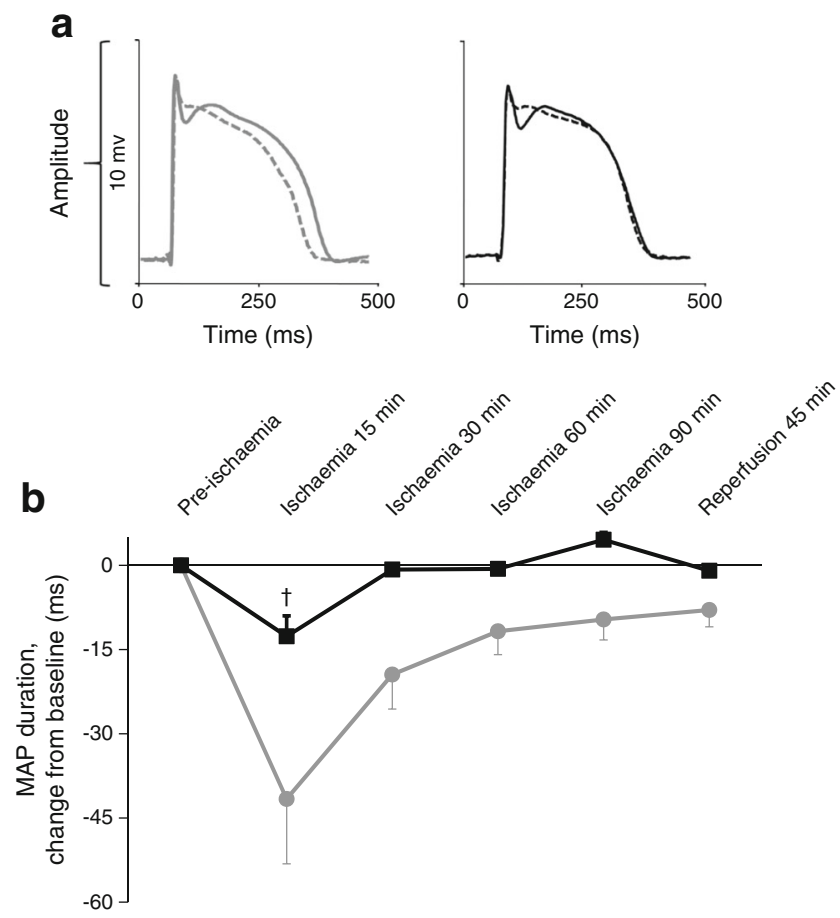

Fig. 2 MAP duration in ischaemia and reperfusion. (a) Representative MAPs recorded from the epicardium in the central portion of the ischaemic zone prior to ischaemia (solid lines) and at $15 \mathrm{~min}$ of low-flow ischaemia (dashed lines) in an untreated pig (left, in grey) and a pig that had received chronic treatment with metformin (right, in black). In the absence of metformin treatment, ischaemia caused shortening of action potential duration. This effect was attenuated by chronic treatment with metformin. (b) MAP duration recorded from the epicardium in the ischaemic region at baseline and during $90 \mathrm{~min}$ of low-flow ischaemia and $45 \mathrm{~min}$ of reperfusion ( $n=13$ in each group). The nadir of action potential duration in untreated pigs (grey) and the most pronounced effect of metformin (black) to prevent action potential shortening occurred at 15 min of ischaemia. ${ }^{\dagger} p=0.02$ for difference in action potential duration between groups 
Chronic metformin treatment significantly attenuated MAP shortening during ischaemia (Fig. 2). The effect was most pronounced at $15 \mathrm{~min}$ of ischaemia, when MAP shortened from baseline by $13 \pm 4 \mathrm{~ms}$ (from $310 \pm 8$ to $297 \pm 11 \mathrm{~ms}$ ) in the chronic metformin group compared with $42 \pm 12 \mathrm{~ms}$ (from $305 \pm 7$ to $263 \pm 7 \mathrm{~ms}$ ) in the untreated group ( $p=0.02$ ). MAP duration in the non-ischaemic lateral LV wall did not differ between chronic metformin and untreated groups at baseline (315 \pm 9 and $313 \pm 14 \mathrm{~ms}$, respectively) and was unaffected in either group by ischaemia of the anterior LV. Consequently, at $15 \mathrm{~min}$ of ischaemia the difference in MAP duration between ischaemic and non-ischaemic regions widened to $38 \pm 14 \mathrm{~ms}$ in untreated pigs, but was limited to $9 \pm 4 \mathrm{~ms}$ in the chronic metformin group $(p<0.001)$. Thus, chronic metformin treatment reduced regional heterogeneity of repolarisation during ischaemia.

Acute metformin treatment did not affect MAP duration in the absence of ischaemia (302 $\pm 5 \mathrm{~ms}$ before treatment; $305 \pm 8 \mathrm{~ms} 180 \mathrm{~min}$ after treatment) and did not attenuate MAP shortening with ischaemia (to $278 \pm 12 \mathrm{~ms}$ at $15 \mathrm{~min}$ of ischaemia) compared with untreated controls.

\section{Myocardial ATP concentration during ischaemia}

Shortening of action potential duration during ischaemia is due primarily to opening of $\mathrm{K}_{\mathrm{ATP}}$ channels, which in turn is triggered by acidosis, accumulation of ADP and depletion of ATP [22, 23]. To evaluate whether a lesser degree of action potential shortening during ischaemia was associated with greater myocardial ATP concentration in pigs treated chronically with metformin, ATP was measured in biopsy samples obtained at $15 \mathrm{~min}$ of ischaemia, when action potential shortening was most pronounced. ATP concentration at $15 \mathrm{~min}$ of ischaemia was higher in pigs treated chronically with metformin than in untreated pigs $(4.1 \pm 0.2 \mathrm{vs}$ $3.5 \pm 0.1 \mu \mathrm{mol} / \mathrm{g}, p=0.04$; Fig. 3). This suggests that the action potential stabilising and anti-fibrillatory effects of chronic metformin treatment are due to preserved ATP concentration during ischaemia.

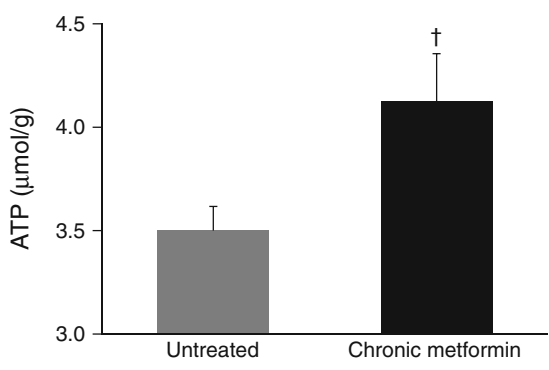

Fig. 3 Effect of chronic metformin treatment on myocardial ATP concentration during ischaemia. Myocardial ATP concentration in drill biopsies obtained at $15 \mathrm{~min}$ of regional myocardial ischaemia in eight pigs that received chronic metformin treatment (black) and seven untreated pigs (grey). ATP concentration was greater in the metformin group. ${ }^{\dagger} p=0.039$
To understand the basis for ATP conservation during ischaemia with chronic metformin treatment, we examined components of energy balance including myocardial contractile function and oxygen and substrate utilisation in the ischaemic zone (Table 1). Indices of regional and global contractile function (regional stroke work index and $\mathrm{LV} \mathrm{dP} / \mathrm{dt}_{\max }$ ) were similar at baseline and declined to a similar degree during ischaemia in both groups. Likewise, myocardial oxygen consumption and uptake of glucose and lactate were similar at baseline and were similarly affected by ischaemia in both groups. During ischaemia, both groups demonstrated comparable decreases in oxygen consumption due to $50 \%$ LAD flow reduction and slight widening of the coronary arteriovenous oxygen difference, increased glucose extraction and transition to net lactate release. In aggregate, these findings imply that differences in myocardial ATP concentration during ischaemia did not arise from differences in contractile function, oxidative metabolism or non-oxidative glycolysis.

Interaction of metformin with $\mathbf{K}_{\mathrm{ATP}}$ channel opener We evaluated the alternative possibility that attenuation of action potential shortening during ischaemia by chronic metformin treatment is due to direct blockade of cardiac $\mathrm{K}_{\mathrm{ATP}}$ channels by metformin. $\mathrm{K}_{\mathrm{ATP}}$ channels are ordinarily closed under non-ischaemic conditions. Accordingly, we infused
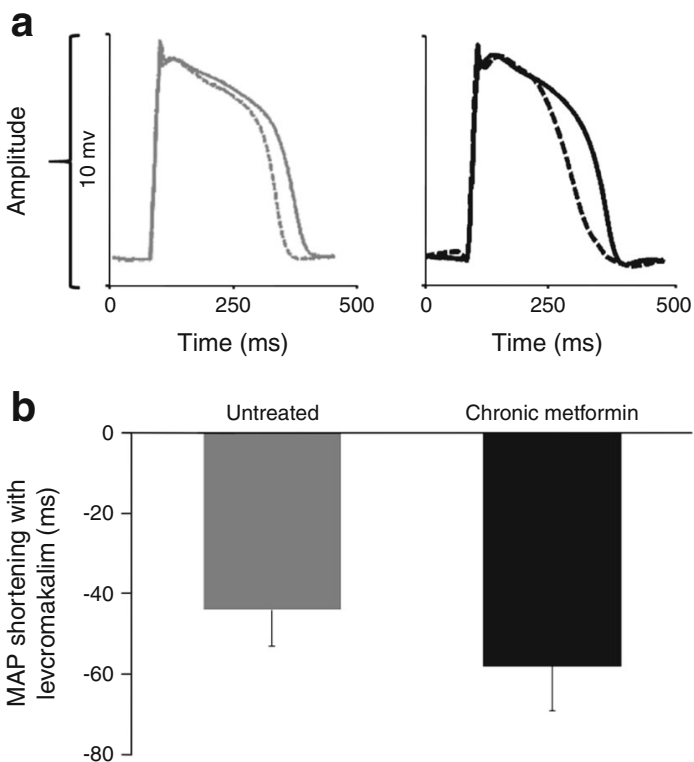

Fig. 4 No effect of chronic metformin treatment on MAP shortening in response to levcromakalim. (a) Representative MAP recordings under non-ischaemic conditions from LV epicardium before (solid lines) and during (dashed lines) infusion of the $\mathrm{K}_{\text {ATP }}$ channel opener levcromakalim $\left(1 \mu \mathrm{g} \mathrm{kg}^{-1} \mathrm{~min}^{-1}\right.$, intracoronary) in an untreated pig (left, grey) and in a pig that received chronic treatment with metformin (right, black). MAP shortens during infusion of levcromakalim; this effect is unaltered by chronic metformin treatment. (b) Group data showing no effect of chronic metformin treatment on the extent of MAP shortening during intracoronary levcromakalim infusion ( $n=8$ in each group) 
levcromakalim, a $\mathrm{K}_{\text {ATP }}$ channel opener, into the LAD under non-ischaemic conditions and determined whether the resulting MAP shortening was attenuated by chronic metformin treatment. In pigs that were not treated with metformin, levcromakalim shortened MAP by $44 \pm 9$ ms (Fig. 4), similar to the magnitude of MAP shortening observed at $15 \mathrm{~min}$ of ischaemia. Chronic metformin treatment had no effect on the response to levcromakalim (MAP shortened by $58 \pm 11 \mathrm{~ms}$; $p=\mathrm{NS}$ compared with untreated controls), indicating that metformin does not directly block $\mathrm{K}_{\mathrm{ATP}}$ channels.

AMPK, p-AMPK-Thr ${ }^{172}$ and CS activity A biochemical signature of metformin action in target tissues is activation of AMPK as indicated by its phosphorylation at $\mathrm{Thr}^{172}$ (p-AMPK) [24]. Prior to ischaemia, total AMPK was slightly greater $(p<0.01$; Fig. 5a) and p-AMPK was substantially greater $(p<0.001$; Fig. $5 b)$ in pigs that received chronic metformin treatment compared with untreated controls. At 15 min of ischaemia, p-AMPK increased from pre-ischaemic levels in both groups (main effect of ischaemia; $p<0.001$ ), but

\section{a}
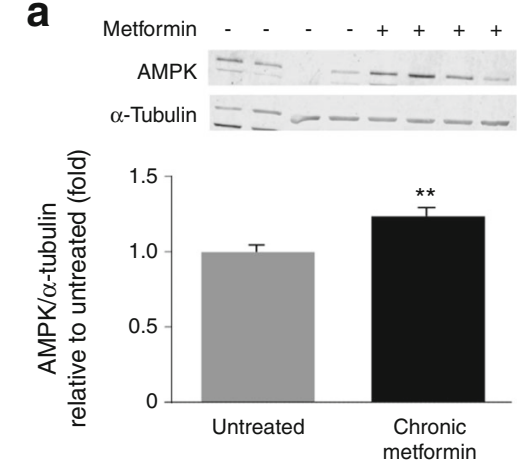

C
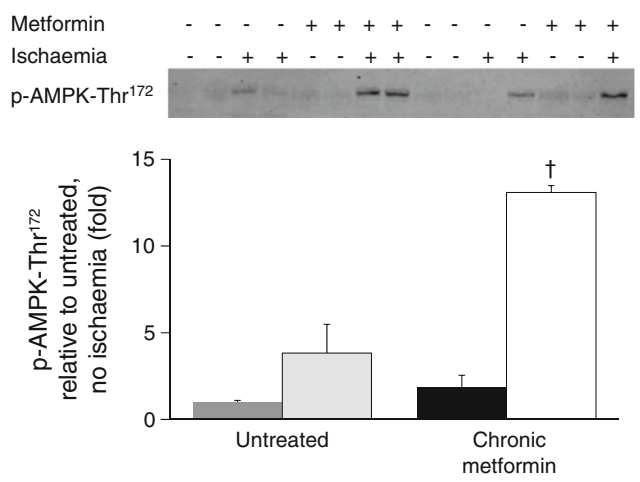

Fig. 5 Effect of metformin treatment on AMPK, p-AMPK-Thr ${ }^{172}$ and CS activity. Example western blot and aggregate data for total AMPK content (a) and p-AMPK-Thr ${ }^{172}$ content (b) in non-ischaemic myocardium of pigs treated chronically with metformin (black, $n=8$ ) and untreated controls (grey, $n=8$ ). ** $p<0.01 ; * * * p<0.001$. (c) A subset of the pigs depicted in (b) underwent a second myocardial biopsy at $15 \mathrm{~min}$ of ischaemia (dark grey, untreated/pre-ischaemia; light grey, untreated/ischaemia; black, chronic metformin/pre-ischaemia; white, chronic metfor$\mathrm{min} /$ ischaemia; $n=3-4$ in each category). Ischaemia and chronic metformin treatment each increased p-AMPK-Thr ${ }^{172}(p<0.001$ for main effect the effect was markedly amplified by chronic metformin treatment $(p=0.002$ for interaction of treatment and ischaemia on p-AMPK; Fig. 5c). Acute metformin treatment did not affect p-AMPK content (post-treatment expression $0.94 \pm 0.04$ of pretreatment expression).

CS was determined as a marker of aerobic metabolic capacity. Prior to ischaemia, myocardial CS activity was lower in metformin-treated pigs than in untreated pigs $(p<0.001)$. During ischaemia, CS activity declined in untreated pigs $(p=0.03)$, but not in metformin-treated pigs. There was a significant interaction of treatment and ischaemia on CS activity ( $p=0.02$; Fig. $5 \mathrm{~d})$.

\section{Discussion}

Death from ischaemic VF was prevented when normal pigs were pretreated with metformin for 2-3 weeks at a clinically relevant dose. The findings indicate a plausible mechanism by which metformin may reduce cardiac mortality in humans,

b
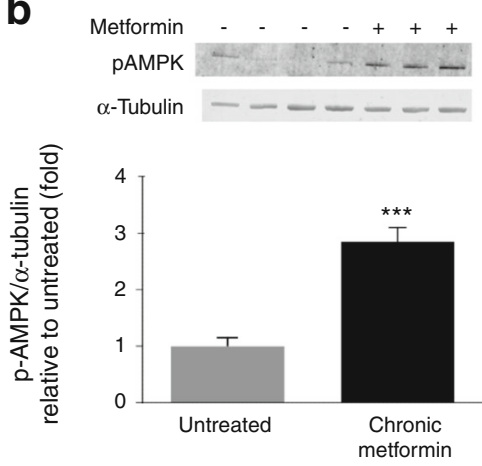

\section{d}

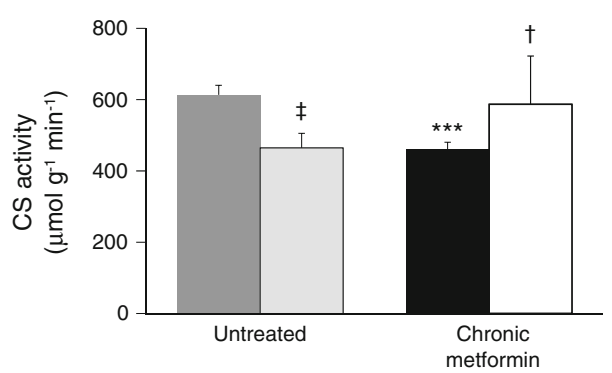

of each factor), with significant interaction of ischaemia and metformin treatment $\left({ }^{\dagger} p=0.002\right)$. (d) CS activity in biopsy samples from untreated pigs (dark grey, no ischaemia, $n=13$; light grey, $15 \mathrm{~min}$ of ischaemia, $n=3$ ) and from pigs treated chronically with metformin (black, no ischaemia, $n=8$; white, ischaemia, $n=4$ ). Under non-ischaemic conditions, CS activity was lower in metformin-treated pigs compared with untreated controls $(* * * p<0.001)$. With ischaemia, CS activity decreased in untreated pigs $\left({ }^{\ddagger} p=0.03\right)$, but not in metformin-treated pigs $\left({ }^{\dagger} p=0.02\right.$ for interaction of treatment and ischaemia) 
and suggest that such a benefit might not depend upon the presence of an underlying metabolic abnormality such as diabetes.

The protective metabolic and electrophysiological effects of chronic metformin treatment are shown in Fig. 6. Prior to ischaemia, a myocardial effect of metformin was reflected by activation of AMPK (phosphorylation at $\mathrm{Thr}^{172}$ ) as previously observed in non-diabetic mice [25] and believed to be due to a modest increase in AMP concentration from weak inhibition of complex I of the electron transport chain [26]. Ischaemia is also known to activate AMPK, with a maximum effect approximately $15 \mathrm{~min}$ after ischaemia onset [27]. In the current experiments, metformin amplified the effect of ischaemia on p-AMPK, resulting in a marked augmentation of p-AMPK at 15 min of ischaemia in metformin-treated hearts. We demonstrate an association of AMPK-activating and anti-fibrillatory effects of metformin, which does not prove causality. However, there is considerable evidence to support this possibility. AMPK serves to maintain cellular

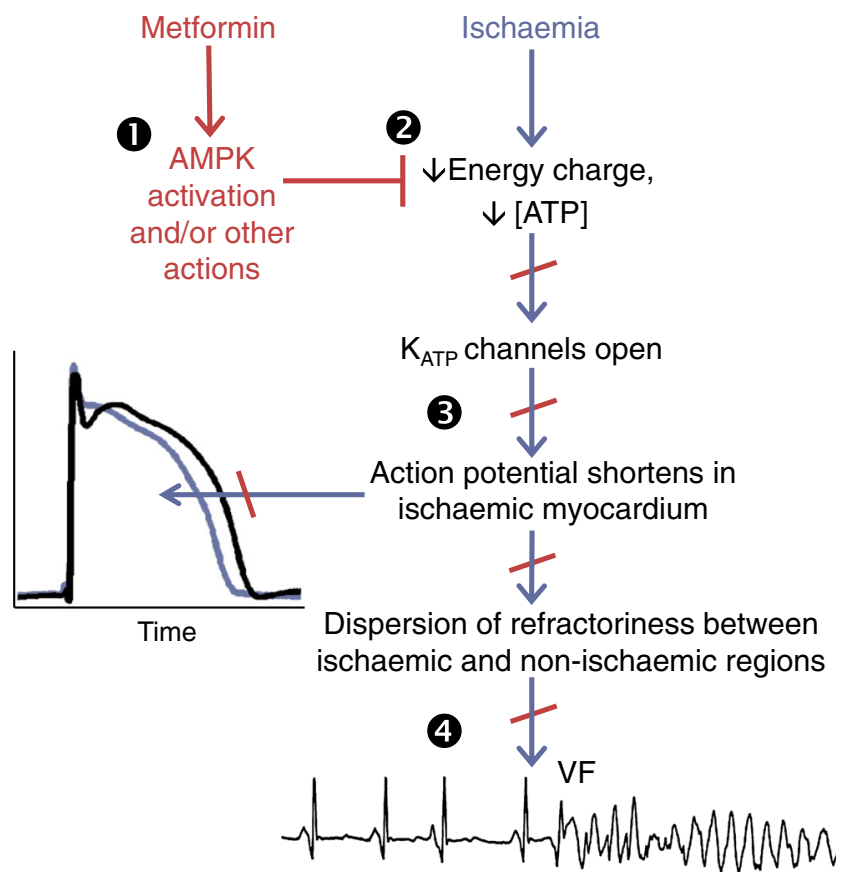

Fig. 6 Proposed mechanism of protection from ischaemic VF by chronic metformin treatment. Effects of ischaemia are indicated in blue; effects of chronic metformin treatment are indicated in red. Ordinarily, ischaemia causes a decline in myocardial energy charge and ATP concentration, leading $\mathrm{K}_{\mathrm{ATP}}$ channels to open and action potential duration to shorten. In non-ischaemic myocardium, action potential duration does not change. Thus, ischaemia increases the dispersion of repolarisation and refractoriness between ischaemic and non-ischaemic regions, predisposing to VF. Chronic metformin treatment increases AMPK activity in normal porcine myocardium, amplifies the activation of AMPK by myocardial ischaemia, and may exert other favourable cellular actions (1). Consequently, chronic metformin treatment results in maintenance of a higher myocardial ATP concentration during ischaemia (2). This limits the degree of ischaemic action potential shortening and dispersion of repolarisation (3), thereby reducing the propensity for $\mathrm{VF}$ compared with untreated pigs (4) energetics under stress through a variety of mechanisms including stimulation of substrate catabolism, inhibition of anabolic pathways, promotion of autophagy and prevention of oxidative stress [28]. In rodents, the consequences of activating AMPK during ischaemia include preserved mitochondrial function [29] and higher ATP levels [30, 31].

Prior to ischaemia, CS activity was lower in the hearts of metformin-treated pigs than in those of untreated controls. However, during ischaemia the reduction in CS activity observed in the hearts of untreated controls was prevented by metformin treatment. Both findings are similar to those previously reported in rat liver [32]. The mechanism for a baseline reduction in CS activity with metformin is uncertain, but it may reflect a coordinated downregulation of electron transport chain complex I and tricarboxylic acid activities [33]. An effect of metformin to preserve CS activity during ischaemia has been observed in rat hearts and liver and may be related to concurrent activation of AMPK [32, 34].

We demonstrate that chronic treatment with metformin allows higher myocardial ATP concentration to be maintained during early ischaemia, when the risk of ischaemic VF is highest. Although the preservation of ATP concentration coincided with augmented activation of AMPK, we cannot exclude the possibility that AMPK-independent effects of metformin contributed to its favourable energetic effects during ischaemia. Maintenance of ATP concentration during ischaemia likely contributed to the anti-fibrillatory effect of chronic metformin treatment by preventing action potential shortening in ischaemic myocardium. Ordinarily, the action potential shortens in response to ischaemia because myocardial $\mathrm{K}_{\mathrm{ATP}}$ channels open as ATP, or the ATP/ADP ratio declines [35]. The propensity for ischaemic VF is increased by regional heterogeneity of repolarisation and refractoriness $[36,37]$ resulting from action potential duration shortening in ischaemic, but not in non-ischaemic, regions. Conversely, when a higher ATP concentration is maintained in ischaemic myocardium, the probability of $\mathrm{K}_{\mathrm{ATP}}$ channel opening is reduced, the degree of action potential shortening in ischaemic regions is attenuated, and heterogeneity of repolarisation and refractoriness is diminished. The alternative explanation that metformin prevented action potential shortening by directly blocking $\mathrm{K}_{\text {ATP }}$ channels is unlikely because chronic metformin treatment did not antagonise the effects of a pharmacological $\mathrm{K}_{\text {ATP }}$ opener (levcromakalim) on action potential duration under non-ischaemic conditions. The absence of a direct $\mathrm{K}_{\mathrm{ATP}}$ channel blocking effect of metformin has also been confirmed in in vitro experiments [38].

The ATP-conserving action of chronic metformin treatment in ischaemia was not due to reduced contractile function, nor to increased glycolytic or oxidative metabolism. We infer the latter two from the absence of a detectable effect of chronic metformin treatment on myocardial glucose uptake, lactate production or oxygen consumption during ischaemia. 
Rather, the ATP-conserving effect of chronic metformin treatment may reflect enhanced mitochondrial efficiency during ischaemia (i.e. increased ATP production per mole of $\mathrm{O}_{2}$ consumption) as observed with metformin treatment in skeletal muscle under metabolic stress [39]. Alternatively, it is possible that activation of AMPK by metformin decreased ATP utilisation for anabolic processes [28].

We found no effect of acute metformin treatment on p-AMPK, action potential dynamics or VF during ischaemia of the porcine heart. By contrast, acute metformin treatment has been shown to activate myocardial AMPK and reduce ischaemic injury in rodent models [25]. The differences could be related to species or to time from treatment measurement ischaemia. Nonetheless, the present findings suggest that a clinical benefit of metformin in myocardial ischaemia is likely to require chronic treatment and is less likely to result from acute treatment in the emergency department or catheterisation laboratory.

Strengths and limitations Strengths of the study include novel insights into the electrophysiological actions of metformin at a clinically relevant dose in a large animal model. Limitations include potential effects of anaesthesia and uncertainty regarding the mechanism by which metformin activates AMPK in the heart, and whether such activation of AMPK is causally related to the ischaemic protection metformin provides.

Opportunities for future research To determine whether effects of metformin on VF depend on AMPK activation, co-treatment with metformin and an AMPK inhibitor might be informative. A reduction of resting coronary blood flow by $50 \%$, as produced in these experiments, requires a restriction of epicardial coronary artery luminal diameter of approximately $95 \%$ [40] (i.e. subtotal coronary stenosis) and results in an approximately $75 \%$ reduction in subendocardial blood flow $[14,16]$. However, individuals with ischaemic VF in acute myocardial infarction may have complete coronary occlusion resulting from coronary thrombosis. Further studies of metformin in a porcine model of complete coronary occlusion may have additional clinical relevance.

Conclusions Metformin is an inexpensive, safe and widely used drug to treat people with type 2 diabetes, and may reduce mortality in that condition. The potential for expanded therapeutic use of metformin to reduce cardiovascular events in people without diabetes is being investigated in large, ongoing clinical trials $[41,42]$. Here we show that metformin prevents ischaemic VF in metabolically normal, non-diabetic pigs. The anti-fibrillatory effect is likely related to preservation of ATP during ischaemia, thereby limiting action potential shortening and the development of regionally heterogeneous repolarisation. Based on the current findings, it is possible that people without diabetes who have, or are at risk of, ischaemic heart disease might be protected from sudden cardiac death by metformin.

Acknowledgements The authors appreciate the contributions of $\mathrm{M}$. Kusumah, surgical/anaesthesia assistant at the Denver VA Medical Center.

Data availability All data generated or analysed during this study are included in this published article (and its ESM files).

Funding GGS and RLS are supported by National Institutes of Health grants 5R01HL049944 and 4T32HL007171. GGS, JEBR and CRG are supported by the Medical Research Service of the US Department of Veterans Affairs. JEBR is supported by the University of Colorado Center for Women's Health.

Duality of interest The authors declare that there is no duality of interest associated with this manuscript.

Contribution statement All authors fulfilled ICMJE uniform requirements for manuscripts submitted to medical journals based upon substantial contributions to conception and design or acquisition and interpretation of data, drafting the article or critically revising it for intellectual content, and provision of approval of the submitted work. GGS is the guarantor of this work.

\section{References}

1. UK Prospective Diabetes Study (UKPDS) Group (1998) Effect of intensive blood-glucose control with metformin on complications in overweight patients with type 2 diabetes (UKPDS 34). Lancet 352:854-865

2. Abualsuod A, Rutland JJ, Watts TE, Pandat S, Delongchamp R, Mehta JL (2015) The effect of metformin use on left ventricular ejection fraction and mortality post-myocardial infarction. Cardiovasc Drugs Ther 29:265-275

3. Bannister CA, Holden SE, Jenkins-Jones S et al (2014) Can people with type 2 diabetes live longer than those without? A comparison of mortality in people initiated with metformin or sulphonylurea monotherapy and matched, non-diabetic controls. Diabetes Obes Metab 16:1165-1173

4. Lamanna C, Monami M, Marchionni N, Mannucci E (2011) Effect of metformin on cardiovascular events and mortality: a metaanalysis of randomized clinical trials. Diabetes Obes Metab 13: 221-228

5. Roussel R, Travert F, Pasquet B et al (2010) Metformin use and mortality among patients with diabetes and atherothrombosis. Arch Intern Med 170:1892-1899

6. Rena G, Pearson ER, Sakamoto K (2013) Molecular mechanism of action of metformin: old or new insights? Diabetologia 56:18981906

7. Lexis CP, van der Horst IC, Lipsic E et al (2014) Effect of metformin on left ventricular function after acute myocardial infarction in patients without diabetes: the GIPS-III randomized clinical trial. JAMA 311:1526-1535

8. Preiss D, Lloyd SM, Ford I et al (2014) Metformin for non-diabetic patients with coronary heart disease (the CAMERA study): a randomised controlled trial. Lancet Diabetes Endocrinol 2:116-124 
9. Gundewar S, Calvert JW, Jha S et al (2009) Activation of AMPactivated protein kinase by metformin improves left ventricular function and survival in heart failure. Circ Res 104:403-411

10. Ashour AE, Sayed-Ahmed MM, Abd-Allah AR et al (2012) Metformin rescues the myocardium from doxorubicin-induced energy starvation and mitochondrial damage in rats. Oxidative Med Cell Longev 2012:434195

11. Hale SL, Alker KJ, Lo HM, Ingwall JS, Kloner RA (1985) Alterations in the distribution of high-energy phosphates during ischemia in a canine model of reperfusion-induced ventricular fibrillation. Am Heart J 110:590-594

12. Luqman N, Sung RJ, Wang CL, Kuo CT (2007) Myocardial ischemia and ventricular fibrillation: pathophysiology and clinical implications. Int J Cardiol 119:283-290

13. Huang JV, Lu L, Ye S et al (2013) Impaired contractile recovery after low-flow myocardial ischemia in a porcine model of metabolic syndrome. Am J Physiol Heart Circ Physiol 304:H861-H873

14. Xu Y, Gen M, Lu L et al (2005) PPAR-gamma activation fails to provide myocardial protection in ischemia and reperfusion in pigs. Am J Physiol Heart Circ Physiol 288:H1314-H1323

15. Lu L, Xu Y, Greyson CR, Ursell PC, Schwartz GG (1999) Nonelastic deformation of myocardium in low-flow ischemia and reperfusion: ultrastructure-function relations. J Mol Cell Cardiol 31: 1157-1169

16. Schwartz GG, Xu Y, Greyson C, Cohen J, Lu L (1996) Low-dose inotropic stimulation during left ventricular ischaemia does not worsen post-ischaemic dysfunction. Cardiovasc Res 32:1024-1037

17. Franz MR, Flaherty JT, Platia EV, Bulkley BH, Weisfeldt ML (1984) Localization of regional myocardial ischemia by recording of monophasic action potentials. Circulation 69:593-604

18. Lu L, Reiter MJ, Xu Y, Chicco A, Greyson CR, Schwartz GG (2008) Thiazolidinedione drugs block cardiac $\mathrm{K}_{\mathrm{ATP}}$ channels and may increase propensity for ischaemic ventricular fibrillation in pigs. Diabetologia 51:675-685

19. Greyson C, Garcia J, Mayr M, Schwartz GG (1995) Effects of inotropic stimulation on energy metabolism and systolic function of ischemic right ventricle. Am J Phys 268:H1821-H1828

20. Spinazzi M, Casarin A, Pertegato V, Ermani M, Salviati L, Angelini C (2011) Optimization of respiratory chain enzymatic assays in muscle for the diagnosis of mitochondrial disorders. Mitochondrion 11:893-904

21. Keller AC, Knaub LA, Miller MW, Birdsey N, Klemm DJ, Reusch JE (2015) Saxagliptin restores vascular mitochondrial exercise response in the Goto-Kakizaki rat. J Cardiovasc Pharmacol 65:137147

22. Bethell HW, Vandenberg JI, Smith GA, Grace AA (1998) Changes in ventricular repolarization during acidosis and low-flow ischemia. Am J Phys 275:H551-H561

23. Nakaya H (2014) Role of ATP-sensitive K+ channels in cardiac arrhythmias. J Cardiovasc Pharmacol Ther 19:237-243

24. Meng S, Cao J, He Q et al (2015) Metformin activates AMPactivated protein kinase by promoting formation of the alphabetagamma heterotrimeric complex. J Biol Chem 290:37933802

25. Calvert JW, Gundewar S, Jha S et al (2008) Acute metformin therapy confers cardioprotection against myocardial infarction via AMPK-eNOS-mediated signaling. Diabetes 57:696-705
26. Dolinsky VW, Dyck JR (2006) Role of AMP-activated protein kinase in healthy and diseased hearts. Am J Physiol Heart Circ Physiol 291:H2557-H2569

27. Omar MA, Fraser H, Clanachan AS (2008) Ischemia-induced activation of AMPK does not increase glucose uptake in glycogenreplete isolated working rat hearts. Am J Physiol Heart Circ Physiol 294:H1266-H1273

28. Bairwa SC, Parajuli N, Dyck JR (2016) The role of AMPK in cardiomyocyte health and survival. Biochim Biophys Acta 1862: 2199-2210

29. Qi D, Young LH (2015) AMPK: energy sensor and survival mechanism in the ischemic heart. Trends Endocrinol Metab 26:422-429

30. Kawabata H, Ishikawa K (2003) Cardioprotection by metformin is abolished by a nitric oxide synthase inhibitor in ischemic rabbit hearts. Hypertens Res 26:107-110

31. Kim AS, Miller EJ, Wright TM et al (2011) A small molecule AMPK activator protects the heart against ischemia-reperfusion injury. J Mol Cell Cardiol 51:24-32

32. Cahova M, Palenickova E, Dankova H et al (2015) Metformin prevents ischemia reperfusion-induced oxidative stress in the fatty liver by attenuation of reactive oxygen species formation. Am J Physiol Gastrointest Liver Physiol 309:G100-G111

33. Williamson JR, Ford C, Illingworth J, Safer B (1976) Coordination of citric acid cycle activity with electron transport flux. Circ Res 38 : I39-I51

34. Barreto-Torres G, Parodi-Rullan R, Javadov S (2012) The role of PPARalpha in metformin-induced attenuation of mitochondrial dysfunction in acute cardiac ischemia/reperfusion in rats. Int $\mathrm{J}$ Mol Sci 13:7694-7709

35. Saito T, Sato T, Miki T, Seino S, Nakaya H (2005) Role of ATPsensitive $\mathrm{K}^{+}$channels in electrophysiological alterations during myocardial ischemia: a study using Kir 6.2-null mice. Am J Physiol heart Circ Physiol 288:H352-H357

36. Farid TA, Nair K, Masse $\mathrm{S}$ et al (2011) Role of $\mathrm{K}_{\text {ATP }}$ channels in the maintenance of ventricular fibrillation in cardiomyopathic human hearts. Circ Res 109:1309-1318

37. Picard S, Rouet R, Ducouret P et al (1999) $\mathrm{K}_{\mathrm{ATP}}$ channels and 'border zone' arrhythmias: role of the repolarization dispersion between normal and ischaemic ventricular regions. Br J Pharmacol 127:1687-1695

38. Aziz Q, Thomas A, Khambra T, Tinker A (2010) Phenformin has a direct inhibitory effect on the ATP-sensitive potassium channel. Eur J Pharmacol 634:26-32

39. Vytla VS, Ochs RS (2013) Metformin increases mitochondrial energy formation in L6 muscle cell cultures. J Biol Chem 288:2036920377

40. Gould KL, Lipscomb K, Hamilton GW (1974) Physiologic basis for assessing critical coronary stenosis. Instantaneous flow response and regional distribution during coronary hyperemia as measures of coronary flow reserve. Am J Cardiol 33:87-94

41. VA Office of Research and Development (2016) Investigation of metformin in pre-diabetes on atherosclerotic cardiovascular outcomes (VA-IMPACT). Available from www.clinicaltrials.gov. Accessed 20 Feb 2017

42. Cambridge University Hospitals, NHS Foundation Trust, and the University of Cambridge (2014) Glucose lowering in non-diabetic hyperglycaemia trial (GLINT). Available from www. clinicaltrialsregister.eu. Accessed 20 Feb 2017 